\title{
Modafinil improves daytime sleepiness in patients with mild to moderate obstructive sleep apnoea not using standard treatments: a randomised placebo-controlled crossover trial
}

\author{
Julia L Chapman, ${ }^{1,2}$ Liora Kempler, ${ }^{1,2}$ Catherina L Chang, ${ }^{1,2}$ Shaun C Williams, ${ }^{1,2}$ \\ Sheila Sivam, 1,2,3 Keith K H Wong, ${ }^{1,2,3}$ Brendon J Yee, ${ }^{1,2,3}$ Ronald R Grunstein, 1,2,3 \\ Nathaniel S Marshall ${ }^{1,2,4}$
}

\begin{abstract}
${ }^{1}$ The NHMRC Centre for Integrated Research and Understanding of Sleep (CIRUS), The University of Sydney, Sydney, New South Wales, Australia

${ }^{2}$ The Woolcock Institute of Medical Research, Sydney, New South Wales, Australia ${ }^{3}$ Department of Respiratory and Sleep Medicine, Royal Prince Alfred Hospital, Sydney, New South Wales, Australia ${ }^{4}$ Sydney Nursing School, The University of Sydney, Sydney, New South Wales, Australia
\end{abstract}

\section{Correspondence to} Dr Nathaniel Marshall, Sydney Nursing School, University of Sydney, Blg MO2, University of Sydney, Sydney, NSW 2006, Australia; nathaniel.marshall@sydney. edu.au

Received 26 April 2013 Revised 17 September 2013 Accepted 31 October 2013 Published Online First 28 November 2013

\section{CrossMark}

To cite: Chapman JL, Kempler $\mathrm{L}$, Chang $\mathrm{CL}$, et al. Thorax 2014;69:274-279.

\begin{abstract}
Introduction Patients with mild to moderate obstructive sleep apnoea (OSA) commonly suffer excessive daytime sleepiness. Continuous positive airway pressure (CPAP) has limited effectiveness in reducing sleepiness in milder OSA. Modafinil is a wake-promoting drug licensed to treat residual sleepiness in CPAP-treated OSA. We hypothesised that modafinil may effectively treat sleepiness in untreated mild to moderate OSA. Methods Untreated sleepy men with mild to moderate OSA (age 18-70, apnoea-hypopnoea index (AHI) 5-30/h, Epworth Sleepiness Scale (ESS) $\geq 10$ ) were randomised to receive $200 \mathrm{mg}$ modafinil or matching placebo daily for 2 weeks before crossing over to the alternative treatment after a minimum 2-week washout. Mixed model analysis of variance was used to compare the changes on modafinil to placebo while classifying all randomised patients as random factors.

Results 32 patients were randomised (mean (SD) AHI $13(6.4) / h$, age 47 (10.7) years, ESS 13.6 (3.3), body mass index $\left.28.2(3.6) \mathrm{kg} / \mathrm{m}^{2}\right), 29$ of whom $(91 \%)$ completed the trial. The primary outcome (ESS) improved more on modafinil than placebo (3.6 points, $95 \% \mathrm{Cl} 1.3$ to $5.8, p=0.003$ ) and the secondary outcome (40-min driving simulator performance) also improved more on modafinil than placebo (steering deviation $4.7 \mathrm{~cm}, 95 \%$ $\mathrm{Cl} 0.8$ to $8.5, \mathrm{p}=0.018$ ). Psychomotor Vigilance Task reciprocal reaction time improved significantly over placebo $(0.15(1 / \mathrm{ms}), 95 \% \mathrm{Cl} 0.03$ to $0.27, \mathrm{p}=0.016)$. Improvements on the Functional Outcomes of Sleep Questionnaire were not significant (5.3 points over placebo, $95 \% \mathrm{Cl}-1$ to $11.6, \mathrm{p}=0.093)$.

Conclusions Modafinil significantly improved subjective sleepiness in patients with untreated mild to moderate OSA. The size of this effect is clinically relevant at 3-4 ESS points of improvement compared with only 1-2 points in CPAP clinical trials. Driving simulator performance and reaction time also improved on modafinil.
\end{abstract}

Clinical Trial Registration ACTRN\#12608000128392.

\section{INTRODUCTION}

Obstructive sleep apnoea (OSA), defined as an apnoea-hypopnoea index (AHI) of $>5$, affects up to one in five adults. ${ }^{1}$ It is associated with excessive daytime sleepiness and a reduction in cognitive

\section{Key messages}

What is the key question?

- Patients with mild to moderate sleep apnoea often have significant daytime sleepiness that is minimally reduced by CPAP treatment.

What is the bottom line?

- Two weeks of treatment with the wake-promoting drug modafinil (200 mg daily) markedly improved patient sleepiness compared with placebo.

\section{Why read on?}

- Patients with significant sleepiness but mild sleep apnoea on a sleep study are a common conundrum in sleep medicine. Direct pharmacotherapy for the sleepiness may provide greater symptomatic relief than other currently available therapy.

function and quality of life. Severe sleep apnoea has been strongly associated with increased mortality or cardiovascular disease, but the prognosis for people with milder OSA is much less clear and may not differ from people without OSA. ${ }^{2-5}$

Excessive daytime sleepiness (Epworth Sleepiness Scale (ESS) $>10)^{6}$ is present in about $28 \%$ of people with mild to moderate sleep apnoea (AHI 5-29). ${ }^{7}$ However, both daytime sleepiness ${ }^{8}$ and sleep apnoea are common, ${ }^{9}$ so some of the people suffering from both conditions may do so by chance, or possibly because of the effects of underlying obesity or depression. ${ }^{10}$ Continuous positive airway pressure (CPAP) is considered the 'gold standard' treatment for severe OSA, ${ }^{11}$ yet the effect of CPAP on parameters of daytime sleepiness in patients with mild to moderate OSA is limited, showing a decrease of 1-2 points in the ESS and a limited number of patients (19\%) with normalised scores (ESS <10) after treatment. ${ }^{12}{ }^{13}$ Adherence to CPAP is also problematic with $46-83 \%$ of patients unable to maintain therapy. ${ }^{14}$

Modafinil is a wakefulness promoter that has been approved for use in Australia, the USA and Europe for the treatment of excessive daytime sleepiness associated with narcolepsy, shiftwork 
sleep disorder and also for residual daytime sleepiness in patients with OSA despite adequate CPAP use. ${ }^{15}{ }^{16}$ In Europe only the indication for narcolepsy remains following a 2011 review (http://www.ema.europa.eu). Modafinil successfully reduces residual daytime sleepiness in patients with OSA using CPAP, an effect shown in multiple clinical trials. ${ }^{17-21}$ We have previously shown that modafinil increases alertness compared with placebo in patients briefly withdrawn from CPAP. $^{22}$

Given the limited effect of CPAP on sleepiness in mild to moderate OSA and poor compliance with this therapy, it is possible that modafinil may have provide an alternative treatment for symptomatic relief in such patients. We hypothesised that 2 weeks of daily modafinil (200 mg) would significantly improve daytime sleepiness and function compared with placebo in patients with untreated mild to moderate OSA.

\section{METHODS}

Patients meeting the inclusion and exclusion criteria were randomised to receive either placebo or modafinil $200 \mathrm{mg}(2 \times 100 \mathrm{mg}$ capsules every morning) for 2 weeks before crossing over to the alternative treatment after a minimum 2-week washout. Outcome measures were collected at the baseline and end of each treatment period.

\section{Patients}

Patients were included in the study if they met the following criteria: men aged 18-70 years, AHI 5-30/h from overnight in-laboratory polysomnogram (PSG) within the past 6 months (see a description of PSG scoring criteria in a previous study from our research group), ${ }^{23}$ ESS $\geq 10$, no prior use of modafinil or armodafinil, treatment with CPAP or mandibular advancement splint (MAS) within the past 3 months, no psychological disorder or other sleep disorder/shift work, no drug or alcohol abuse, no use of concomitant medications metabolised by cytochrome P450 enzyme CYP3A4, no severe renal or hepatic impairment. All patients underwent a full medical screen by an Australian government-accredited sleep medicine practitioner with $>10$ years of clinical experience. Patients with any history of non-OSA sleep disorder including regular sleep restriction were not entered into the study. Study physicians also excluded patients who clinically presented with driving-related sleepiness that could endanger the public and they were treated immediately outside of the clinical trial.

\section{Study drug}

Patients were randomised to receive modafinil $200 \mathrm{mg}$ ( $2 \times 100 \mathrm{mg}$ capsules) or matching placebo. Modafinil was provided by CSL Biotherapies Ltd in the form of $100 \mathrm{mg}$ white film-coated tablets, which were over-encapsulated with opaque maroon-coloured capsules by unblinded pharmacy staff who also prepared matching placebo capsules.

Randomisation and treatment allocation concealment were achieved by a randomisation sequence that was generated electronically by an independent statistician using a statistical package (SAS) with a block size of four and with a 1:1 ratio. This was generated and given to the pharmacist who dispensed all medication but was never involved in patient selection or outcome measurements. No study clinician, person enrolling a patient or outcomes assessor had knowledge of study drug allocation, so we regard this trial as 'double-blind'.

Patients were dispensed 2 weeks of modafinil or matching placebo at the baseline of each treatment period. They were instructed to orally ingest two capsules daily in the morning at breakfast. Compliance was measured by treatment diary and counting returned medication.

\section{Study procedures}

Patients were recruited from Royal Prince Alfred Hospital and the Woolcock Institute of Medical Research, Sydney, Australia. All patient visits were conducted at the Woolcock Institute of Medical Research between October 2009 and November 2012.

All patients provided written informed consent prior to proceeding with the study. At the screening visit the following measurements were made: fasting blood tests to check for hepatic or renal impairment, medical history, sitting blood pressure, weight and height. The ESS $^{6}$ and Functional Outcomes of Sleep Questionnaire (FOSQ) ${ }^{24}$ were also administered.

Patients who met the study inclusion/exclusion criteria were asked to present to the clinic for a baseline visit at 12:30 on the day of testing. Their blood pressure and weight were recorded, before allowing them a 5 -min practice on the driving simulator (AusEd) ${ }^{25}$ and the Psychomotor Vigilance Task (PVT). ${ }^{26}$ They were asked to complete the ESS and FOSQ questionnaires electronically. Medications and medication diaries were dispensed to start the day after the baseline visit.

The ESS questionnaire asks patients to judge their likelihood of falling asleep in eight given situations. In this study, patients were asked how sleepy they felt over the previous 2 weeks. This questionnaire is given a score out of 24 , with $\geq 10$ used to describe 'excessive' daytime sleepiness. ${ }^{6}$ Improvement in the ESS was the primary outcome of the trial.

The $40 \mathrm{~min}$ AusEd driving simulator began at 14:30 h (Woolcock Institute of Medical Research, Sydney, Australia) ${ }^{25}$ and was used to quantify the ability of patients to accurately undertake a complex but under-stimulating cognitivebehavioural task (ie, driving on a country road at night). The first $4 \mathrm{~min}$ of the 40 -min drive was excluded to allow for patients to acclimatise to the programme and controls which left four 9-min epochs for analysis. Patients were instructed to maintain their speed between 60 and $80 \mathrm{~km} / \mathrm{h}$, to maintain a central position in the left hand lane and to brake as quickly as possible at the appearance of a truck. The single secondary outcome of the trial was from the simulator and was the SD in the median lane position (also called steering deviation). Our tertiary outcomes were the 10-min PVT and the FOSQ.

Behavioural alertness was assessed using the 10-min PVT at 13:00, 14:00 and 16:00 (Ambulatory Monitoring, Ardsley, New York, USA). ${ }^{26}$ The PVT is a hand-held box with a small screen and two response buttons. Visual stimuli (red numbers counting up in milliseconds from zero) appear at variable intervals over the test. Patients were instructed to respond as fast as possible to the appearance of numbers on the screen by clicking a button. Specific outcomes collected from this measurement were the mean reaction times transformed for analysis into the reciprocal of the reaction times in milliseconds $(1 / \mathrm{ms})$ and the number of lapses (reaction time $\geq 500 \mathrm{~ms}$ ) (x) transformed for analysis using the formula $(\sqrt{\mathrm{x}}+\sqrt{(\mathrm{x}+1)})$. Sleepiness-related quality of life was measured using the FOSQ, which is a 30 -item questionnaire asking patients to rate on a scale of 1-4 (1=maximal impact, $4=$ no impact) how their sleepiness has had an impact on daytime functioning. ${ }^{24}$ In this study, patients were instructed to think about the previous 2 weeks while completing the questionnaire.

\section{Safety}

Adverse events were monitored throughout the study for safety by asking patients at each study visit if they had had any 
changes in their health since the last visit. Patients were also asked to contact the study doctor immediately if they noticed any change in their health or if they were required to start new medication. Serious adverse events (hospitalisation, death, lifethreatening condition) were reported to the ethics committee as soon as possible after the event. Blood pressure was measured at each visit using an automatic upper arm blood pressure monitor with a medium or large cuff chosen to best fit the patient's arm (Omron M4, Omron Healthcare, Kyoto, Japan).

\section{Statistical analyses}

Our pretrial power calculation indicated that 36 completing patients were needed to detect a change of 2.5 units in ESS assuming $90 \%$ power and an $\alpha$ of $5 \%$ (so $\mathrm{p}<0.05$ is regarded as significant in this study). Data were analysed on an intention-to-treat basis for efficacy and on a per protocol basis for safety (ie, all randomised were included in efficacy analyses and only those people who actually ingested at least one dose of study medication were included in the analysis of side effects of those medications). ${ }^{27}$ If a patient decided to withdraw early from the study, every effort was made to collect at least the primary outcome data and information on adverse effects from the patient at the normal study time points even when they had ceased taking medication.

All statistical analyses were performed using SAS V.9.3 (SAS Corporation, Cary, North Carolina, USA). Change from baseline to end of treatment in continuous variables (ESS, steering deviation, PVT reciprocals of reaction times and PVT transformed lapses) were analysed using mixed model analysis of variance (PROC MIXED in the SAS program), classifying all randomised patients as random factors and treatment as a fixed factor. In the models investigating steering deviation and PVT variables, we also used the time of day that the data were gathered as a fixed factor. We did not impute missing data in any patient but included them in the mixed model and allowed the model to integrate their data into the overall estimates. One of the assumptions of these models is that the residual values are normally distributed. We checked for this by plotting the residuals for each model in histograms and Q-Q plots and all were tolerably normal. To test whether baseline AHI predicted ESS response to treatment, we added an interaction term between treatment and disease severity (mild vs moderate OSA). Mild or moderate OSA was defined by an AHI cut-off of $15 / \mathrm{h}$. Whether the patient had a side effect on either modafinil or placebo or both was a repeated measure dichotomous variable that was analysed using a generalised estimating equation. McNemar's test was used to assess the dichotomised data of whether or not ESS had normalised. The primary outcome was the change in ESS scores from baseline to end of treatment. The secondary outcome was the change in the SD in the median lane position (also called steering deviation) as measured by the AusEd driving simulator.

\section{RESULTS}

Forty-three patients were screened for the study and 32 met the inclusion criteria and were randomised (figure 1). The study was stopped early after a much longer than anticipated 3-year recruitment period (October 2009-November 2012) during which we were unable to randomise the power-calculated 36 patients. Slower recruitment related to limited resources for recruitment or advertising and the need to exclude patients with cardiovascular comorbidities or medications that could theoretically interact with modafinil. One patient withdrew from the study prior to the administration of study medication. Two

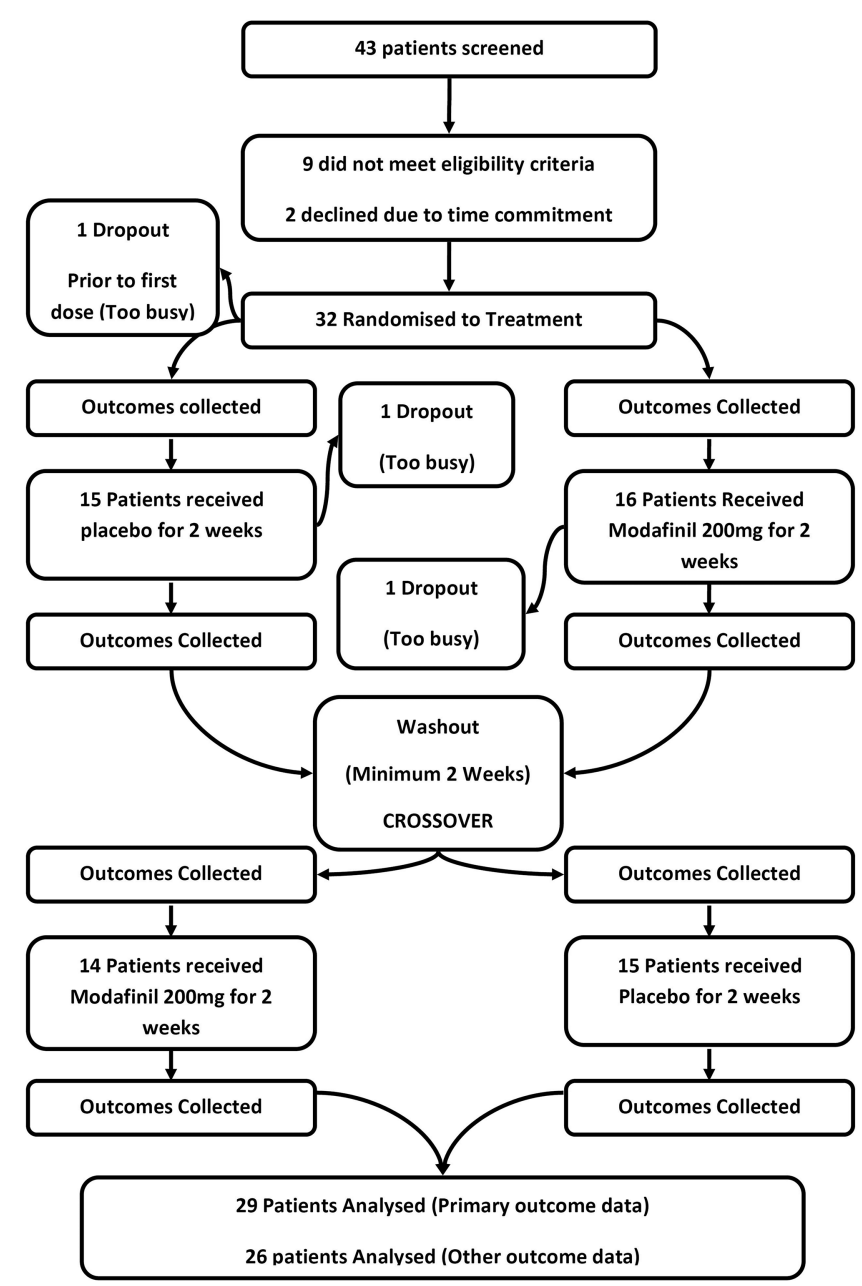

Figure 1 Flowchart of patient recruitment and progression through the trial.

patients withdrew during the study altogether due to inability to complete study visits (one on placebo, one on modafinil, see figure 1).

The primary outcome (ESS) and FOSQ data were collected for 29 of the 32 randomised patients. AusEd and PVT data were collected for 26 patients as three were unable to attend some scheduled study visits. The characteristics of the 32 patients who were randomised are shown in table 1.

ESS improved more on modafinil than placebo (mean net improvement over placebo 3.6 points, 95\% CI 1.3 to 5.8 , $\mathrm{p}=0.003$, figure 2). Two-thirds of the patients had normalised ESS scores $(<10)$ after treatment with modafinil compared with

Table 1 Baseline characteristics of randomised patients

\begin{tabular}{lc}
\hline Patient characteristic & Mean (SD) \\
\hline Age & $47 \pm 10.7$ \\
Apnoea hypopnoea-index & $15.2 \pm 6.4$ \\
Minimum $\mathrm{SpO}_{2}(\%)$ & $86.4 \pm 6.6$ \\
Body mass index $\left(\mathrm{kg} / \mathrm{m}^{2}\right)$ & $28.2 \pm 3.6$ \\
Baseline Epworth score $(/ 24)$ & $13.7 \pm 3.3$ \\
Systolic blood pressure $(\mathrm{mm} \mathrm{Hg})$ & $123 \pm 11.5$ \\
Diastolic blood pressure $(\mathrm{mm} \mathrm{Hg})$ & $80 \pm 9.9$ \\
\hline spo 2 , oxygen saturation. &
\end{tabular}




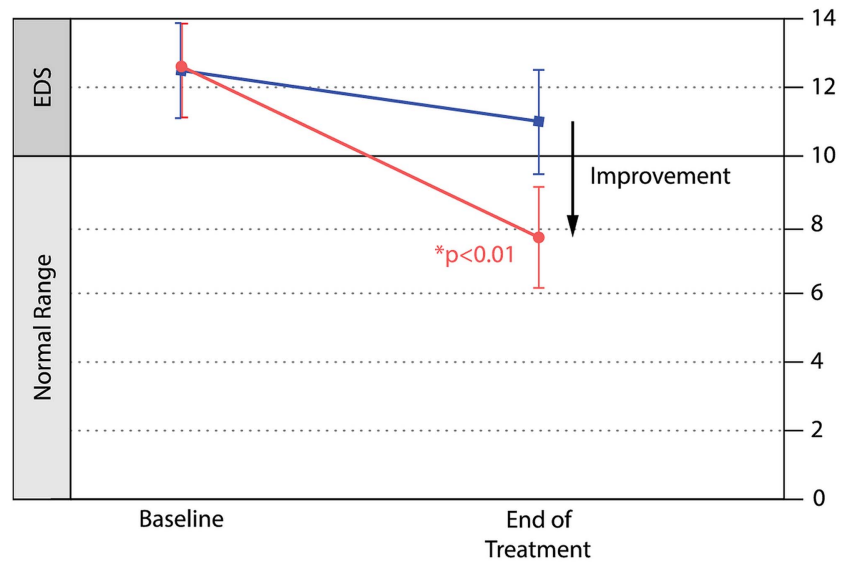

Figure 2 Epworth Sleepiness Scale scores at baseline and at end of treatment. Improvements on modafinil are shown with a red line and circles and placebo with a blue line and squares. The error bars represent the $95 \% \mathrm{Cls}$. The vertical axis gives the score on the Epworth Sleepiness Scale (right) and whether that score is regarded as clinically normal or evidence of excessive daytime sleepiness (EDS). The apparent improvement on modafinil is statistically significant compared with placebo $(p<0.01)$ and moved the patients from being classified as sleepy to being within the normal range on the Epworth Sleepiness Scale.

$33 \%$ after placebo $(\mathrm{S}=0.667, \mathrm{p}=0.414)$. Baseline sleep apnoea severity (mild vs moderate) did not modify the treatment effect (test for interaction $\mathrm{p}=0.108$ ).

The secondary outcome, 40-min AusEd driving simulator performance, improved with modafinil (mean improvement in steering deviation $4.7 \mathrm{~cm}, 95 \%$ CI 0.8 to $8.5, \mathrm{p}=0.018$, figure 3 ). PVT reciprocal reaction time improved more on modafinil than on placebo (mean net effect $0.15(1 / \mathrm{ms}), 95 \%$ CI 0.03 to 0.27 , $\mathrm{p}=0.016$ ). The placebo adjusted effect of modafinil on transformed lapses was a non-significant worsening of 0.26 (95\% CI -0.46 to $0.99, p=0.473)$. The FOSQ total score trended towards a modafinil-induced improvement (mean improvement 5.3 points over placebo, $95 \% \mathrm{CI}-1$ to 11.6 ), but the effect was not statistically significant $(\mathrm{p}=0.093)$.

\section{Safety}

Two patients experienced a serious adverse event of overnight hospitalisation during the trial, both involving cardiovascular disease. One patient with known coronary disease who was in washout after modafinil underwent stent insertion, and the other, who was on placebo at the end of the second treatment arm, suffered ventricular tachycardia. Neither serious adverse event was suspected to be related to study medication.

Four patients reported minor side effects on placebo including dry mouth, headache, anxiety and depression and nausea $($ all $n=1)$. Eleven patients reported minor side effects on modafinil (trouble sleeping $(n=5)$, mood changes $(n=4)$, nausea $(n=2)$, tight cough $(n=1)$, palpitations $(n=1))$. The difference in the number of adverse events between modafinil and placebo was significant (4 patients had side effects on both, 7 on modafinil only, 1 on placebo only and 18 had no side effects); the relative risk for a side effect on modafinil versus placebo was 2.7 (95\% CI 1.4 to $5.3, \mathrm{p}=0.003)$.

There was no significant change in blood pressure between baseline and end of treatment for either systolic (difference between change in systolic blood pressure on modafinil and placebo $0.3 \mathrm{~mm} \mathrm{Hg}, \mathrm{p}=0.921$ ) or diastolic measures (difference between

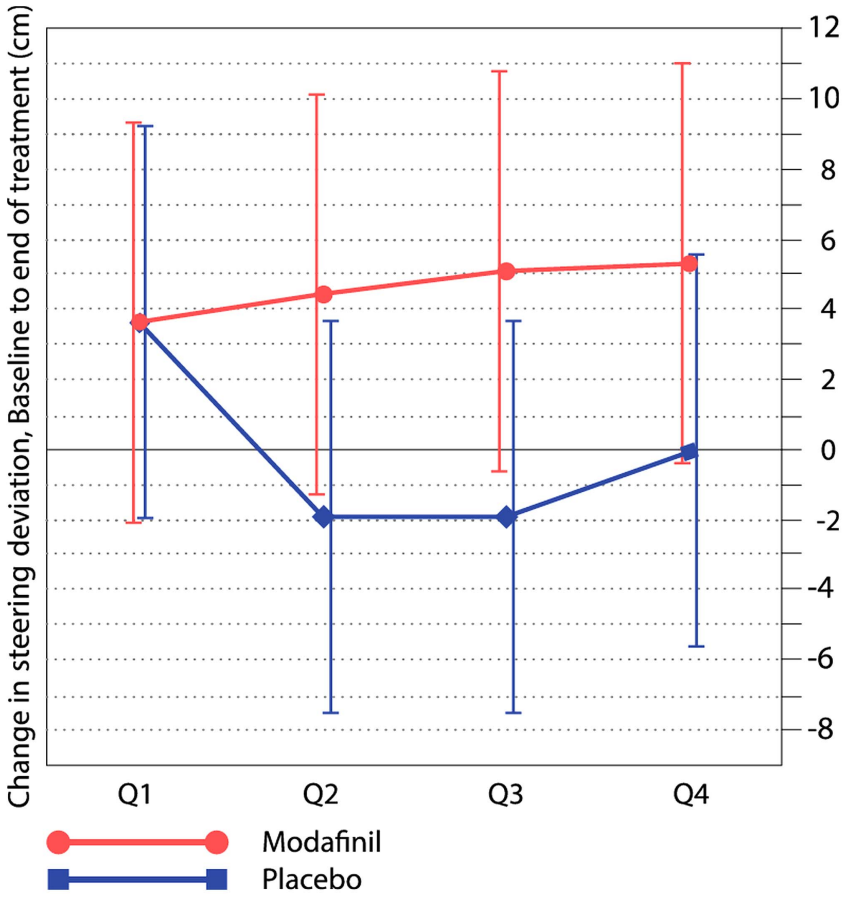

Figure 3 Improvements on modafinil and placebo in steering deviation in the AusEd driving simulator. Improvements on modafinil are shown with a red line and circles and placebo with a blue line and squares. The error bars represent $95 \% \mathrm{Cls}$. The vertical axis gives the improvement in steering deviation in $\mathrm{cm}$ and the horizontal axis shows the period of the drive in quarters of time (Q1 is the first 9 min quarter start of the drive and Q4 is the end 9 min of the drive where fatigue should be highest). ${ }^{31}$ The apparently better performance on modafinil is statistically significant compared with placebo across the quarters $(p<0.01)$ but was not statistically significant when tested within any individual quarter.

change in diastolic blood pressure on modafinil and placebo $1.45 \mathrm{~mm} \mathrm{Hg}, \mathrm{p}=0.493$ ). On modafinil, the systolic blood pressure increased by a mean $\pm S D$ of $2.6 \pm 8.79 \mathrm{~mm} \mathrm{Hg}(\mathrm{p}=0.232)$ and the diastolic blood pressure decreased by $1.8 \pm 9.41 \mathrm{~mm} \mathrm{Hg}$ $(\mathrm{p}=0.309)$.

\section{DISCUSSION}

Modafinil significantly reduced subjective daytime sleepiness as measured by the ESS score (primary outcome) in men with mild to moderate OSA not using other treatments. The improvement in ESS over placebo of 3.6 points (95\% CI 1.3 to 5.8) from a baseline of 13.7 points was non-significantly greater than the decrease of 1.2 points (95\% CI 0.5 to 1.9 ) reported previously in a meta-analysis of the effect of CPAP in patients with mild to moderate OSA who also had average baseline ESS scores of 10-14 points. $^{12}$

The secondary outcome of this trial (simulated driving ability) also improved significantly more on modafinil compared with placebo, as did the tertiary outcome (simple reaction time) with a mean increase in reciprocal reaction time of $0.15(1 / \mathrm{ms})(95 \%$ CI 0.03 to $0.27, \mathrm{p}=0.016)$, which equates to a decrease in real reaction time of $6.6 \mathrm{~ms}$.

The number of lapses on the PVT did not change with either treatment, and sleepiness-related quality of life was nonsignificantly improved (5.3 points, 95\% CI -1 to 11.6, $\mathrm{p}=0.093)$. 
Our study detected a moderate to large clinical effect on the ESS but only small to moderate effects on simulated driving and simple reaction times.

Importantly, the treatment effect size observed in our study may be greater than the two recommended primary treatments for OSA. Previous studies have found that CPAP and MAS reduce ESS by only 1-2 points in similar patients. ${ }^{28} 29$ We have shown that modafinil reduces ESS by about 3.6 points over placebo. The 95\% CIs reported here overlap with those previously reported in similar patients on $\mathrm{CPAP}^{13}$ or MAS, ${ }^{29}$ indicating that modafinil is not inferior and may be superior to these standard treatments. Only $19 \%$ of patients had normalised ESS scores $(<10)$ after treatment with active $\mathrm{CPAP}^{13}$ yet our study showed that $66 \%$ of patients on modafinil had a normalised ESS score. However, before modafinil can be recommended as a treatment in milder OSA with concomitant sleepiness, data from larger and longer trials are needed. It may be reasonable to use modafinil for symptomatic patients where other treatment may be delayed or refused.

The relative risk of suffering an adverse event on modafinil versus placebo was 2.7 (95\% CI 1.4 to $5.3, \mathrm{p}=0.0003)$. These adverse events were primarily transient and mild and consistent with previous studies of modafinil. ${ }^{30}$ Modafinil did not increase blood pressure in this study, but it is important to note that these patients were normotensive at baseline and this finding cannot be extrapolated to a hypertensive population. With a limited number of patients and short trial duration, further studies are necessary to refine this finding in this patient group.

\section{Limitations of the study}

This study is limited by its small and select sample, but the promising results from this study warrant larger studies to be conducted. The study population was limited to men in order to alleviate any gender effect on the secondary outcome variable. This study only assessed the effect of modafinil over a very short time period ( 2 weeks); however, other studies using modafinil in patients on CPAP over 12 weeks show that it is well tolerated over a longer period. ${ }^{17}$ Further research is warranted on a larger number of men and women with untreated OSA using modafinil over a longer period of time.

Another limitation is that we used a 40-min driving simulator. Recent research suggests that people with OSA perform particularly poorly on a longer drive $(90 \mathrm{~min})$. The use of a longer drive in future studies may therefore show a larger clinical effect, particularly in the last half hour. ${ }^{31}$ This study was stopped when it was four patients short of the target recruitment due to limited resources. This may imply a limited population in whom our trial results apply and that external validity should only be presumed in carefully selected patients. It appears that sleepy patients with mild to moderate OSA are often on medication that could interact with modafinil. It is also possible that eligible patients are not interacting with a sleep medicine referral pathway and so we were unable to contact them. Future triallists might want to use more expansive recruitment strategies.

This study is important as it suggests that there may be a potential alternative to CPAP for patients with mild to moderate sleep apnoea and excessive daytime sleepiness. Partly driven by low adherence, CPAP does not deliver large clinically significant reductions in daytime sleepiness in these patients. Although not proven in this study, modafinil may reduce daytime sleepiness by a greater amount than CPAP and may be easier for patients to use. It should be noted, however, that modafinil does not treat any potential cardiovascular disease risks that may be present in more severely afflicted patients towards the top of the AHI range or those with particularly severe oxygen desaturation events.

\section{CONCLUSION}

Compared with placebo, modafinil significantly improved subjective daytime sleepiness, simulated driving ability and simple reaction times but not sleepiness-related quality of life in patients with mild to moderate OSA and significant daytime sleepiness. The symptomatic benefit reported by patients was at least as good as that seen with the gold standard CPAP or MAS therapies. Routine use of modafinil cannot yet be recommended without larger and longer confirmatory trials.

Acknowledgements The authors would like to thank Corianne Kwan and the staff at the Royal Prince Alfred Pharmacy for their aid in preparation handling and dispensing study medication and Professor Peter Liu for generating the randomisation sequence and for help in designing the protocol. We would also like to thank Brendan Funnell for graphical design assistance.

Contributors JLC: Acquisition of data, analysis and interpretation of data and drafting and final approval of article. LK: Acquisition of data, revision and final approval of article. CLC, SS: Acquisition of data, revision of article, final approval of article. SCW, KKHW, BJY, RRG: Conception and design, acquisition of data, revision of article, final approval of article. NSM: Conception and design, acquisition of data, analysis and interpretation of data, drafting and revising article and final approval of article.

Funding Research supported by an Australian NHMRC grant to NSM (1004528) and a University of Sydney Bridging Grant to NSM and NHMRC grants to RRG (264598, 571421, 457094 and 1022730). JLC is supported by a NHMRC postgraduate scholarship (1038709).

Competing interests Our department has received in-kind support from Teva Cephalon for a different clinical trial for sleep apnoea in the last 24 months. The medication tested in this trial and a previous related trial ${ }^{22}$ was provided free of charge by the Australian distributor of modafinil, CSL Biotherapies Ltd. SCW has been an employee of Novartis since March 2013. His employment there began after data analysis and initial drafting of this manuscript was completed. We have no other conflicts of interest with relation to this investigator-initiated study.

Ethics approval The Sydney Local Health District (Royal Prince Alfred Hospital Zone) Human Research Ethics Committee oversaw the approval of the protocol (Protocol Number: X08-0004-initial approval 2008, X12-0078-reapproval 2012, protocol available from the corresponding author) and the trial was registered with the Australia and New Zealand Clinical Trials Registry before the trial began (ACTRN12608000128392).

Provenance and peer review Not commissioned; externally peer reviewed.

Data sharing statement De-identified dataset available upon request from corresponding author.

\section{REFERENCES}

1 Young T, Peppard PE, Gottlieb DJ. Epidemiology of obstructive sleep apnea: a population health perspective. Am J Respir Crit Care Med 2002;165:1217-39.

2 Marshall NS, Wong KKH, Liu PY, et al. Sleep apnea as an independent risk factor for all-cause mortality: the Busselton Health Study. Sleep 2008;31:1079-85.

3 Punjabi NM, Caffo BS, Goodwin JL, et al. Sleep-disordered breathing and mortality: a prospective cohort study. PLoS Med 2009;6:e1000132.

4 Young T, Finn L, Peppard PE, et al. Sleep disordered breathing and mortality: eighteen-year follow-up of the Wisconsin sleep cohort. Sleep 2008;31:1071-8

5 Marin JM, Carrizo SJ, Vicente E, et al. Long-term cardiovascular outcomes in men with obstructive sleep apnoea-hypopnoea with or without treatment with continuous positive airway pressure: an observational study. Lancet 2005;365:1046-53.

6 Johns MW. A new method for measuring daytime sleepiness: the Epworth sleepiness scale. Sleep 1991;14:540-5.

7 Gottlieb DJ, Whitney CW, Bonekat WH, et al. Relation of sleepiness to respiratory disturbance index: the Sleep Heart Health Study. Am J Respir Crit Care Med 1999:159:502-7.

8 Gander PH, Marshall NS, Harris R, et al. The Epworth Sleepiness Scale: influence of age, ethnicity, and socioeconomic deprivation. Epworth Sleepiness scores of adults in New Zealand. Sleep 2005:28:249-53.

9 Young T, Palta M, Dempsey J, et al. The occurrence of sleep-disordered breathing among middle-aged adults. N Engl J Med 1993;328:1230-5. 
10 Bixler EO, Vgontzas AN, Lin HM, et al. Excessive daytime sleepiness in a general population sample: the role of sleep apnea, age, obesity, diabetes, and depression. J Clin Endocrinol Metab 2005;90:4510-15.

11 Kushida CA, Littner MR, Hirshkowitz M, et al. Practice parameters for the use of continuous and bilevel positive airway pressure devices to treat adult patients with sleep-related breathing disorders. Sleep 2006;29:375-80.

12 Marshall NS, Barnes M, Travier N, et al. Continuous positive airway pressure reduces daytime sleepiness in mild to moderate obstructive sleep apnoea: a meta-analysis. Thorax 2006;61:430-4.

13 Weaver TE, Mancini C, Maislin G, et al. Continuous positive airway pressure treatment of sleepy patients with milder obstructive sleep apnea: results of the CPAP Apnea Trial North American Program (CATNAP) randomized clinical trial. Am J Respir Crit Care Med 2012;186:677-83.

14 Weaver TE, Grunstein RR. Adherence to continuous positive airway pressure therapy: the challenge to effective treatment. Proc Am Thorac Soc 2008;5:173-8.

15 Gasa M, Tamisier R, Launois SH, et al. Residual sleepiness in sleep apnoea patients treated by continuous positive airway pressure. J Sleep Res 2013;22:389-97.

16 Ballon JS, Feifel D. A systematic review of modafinil: potential clinical uses and mechanisms of action. J Clin Psychiatry 2006;67:554-66.

17 Black JE, Hirshkowitz M. Modafinil for treatment of residual excessive sleepiness in nasal continuous positive airway pressure-treated obstructive sleep apnea/hypopnea syndrome. Sleep 2005;28:464-71.

18 Pack Al, Black JE, Schwartz JR, et al. Modafinil as adjunct therapy for daytime sleepiness in obstructive sleep apnea. Am J Respir Crit Care Med 2001:164:1675-81.

19 Dinges DF, Weaver TE. Effects of modafinil on sustained attention performance and quality of life in OSA patients with residual sleepiness while being treated with nCPAP. Sleep Med 2003;4:393-402.

20 Bittencourt LR, Lucchesi LM, Rueda $A D$, et al. Placebo and modafinil effect on sleepiness in obstructive sleep apnea. Prog Neuropsychopharmacol Biol Psychiatry 2008;32:552-9.
21 Kingshott RN, Vennelle M, Coleman EL, et al. Randomized, double-blind, placebo-controlled crossover trial of modafinil in the treatment of residual excessive daytime sleepiness in the sleep apnea/hypopnea syndrome. Am J Respir Crit Care Med 2001;163:918-23.

22 Williams SC, Marshall NS, Kennerson M, et al. Modafinil effects during acute continuous positive airway pressure withdrawal: a randomized crossover double-blind placebo-controlled trial. Am J Respir Crit Care Med 2010;181:825-31.

23 Marshall NS, Yee BJ, Desai AV, et al. Two randomized placebo-controlled trials to evaluate the efficacy and tolerability of mirtazapine for the treatment of obstructive sleep apnea. Sleep 2008;31:824-31.

24 Weaver TE, Laizner AM, Evans LK, et al. An instrument to measure functional status outcomes for disorders of excessive sleepiness. Sleep 1997;20:835-43.

25 Desai AV, Wilsmore B, Bartlett DJ, et al. The utility of the AusEd driving simulator in the clinical assessment of driver fatigue. Behav Res Methods 2007:39:673-81.

26 Dinges DF, Powell JW. Microcomputer analyses of performance on a portable, simple visual RT task during sustained operations. Behav Res Methods $1985 ; 17: 652-5$

27 loannidis JPA, Evans SJW, Gøtzsche PC, et al. Better reporting of harms in randomized trials: an extension of the CONSORT statement. Ann Intern Med 2004;141:781-8.

28 Gotsopoulos H, Chen C, Qian J, et al. Oral appliance therapy improves symptoms in obstructive sleep apnea: a randomized, controlled trial. Am J Respir Crit Care Med 2002:166:743-8.

29 Barnes M, Mcevoy RD, Banks S, et al. Efficacy of positive airway pressure and oral appliance in mild to moderate obstructive sleep apnea. Am J Respir Crit Care Med 2004; 170:656-64.

30 Roth T, Schwartz JR, Hirshkowitz M, et al. Evaluation of the safety of modafinil for treatment of excessive sleepiness. J Clin Sleep Med 2007;15:595-602.

31 Vakulin A, Baulk SD, Catcheside PG, et al. Effects of alcohol and sleep restriction on simulated driving performance in untreated patients with obstructive sleep apnea. Ann Intern Med 2009;151:447-55. 